\title{
Optimization Design of Network Development Platform of University Educational Administration Management System Based on Routing Engine
}

\author{
Huang Lin-wei, ${ }^{1, a}$, Lu Xing-hua $^{1, b}$, Ye Shi-tong ${ }^{1, c}$ \\ ${ }^{1}$ Huali College Guangdong University of Technology, Guangzhou Zengcheng, 511325, P. R. China \\ a31769329@qq.com, ${ }^{\mathrm{b}} 44680189 @ q q . c o m$, c $^{\mathrm{a}}$ yesigeng@163.com
}

Keywords: college educational administration, network, system design, routing engine.

\begin{abstract}
The development of the network communication platform of university teaching management system is researched to improve the efficiency of college educational administration management, and promote the sharing and scheduling of academic resource information. University teaching management platform includes four basic entity objects: goal of educational management, teaching information processing nodes, teaching resources data collection node and network communication and information management platform. In addition, it needs to define the external network, remote task management unit and a user to complete the application of the entire system. In the routing and data forwarding protocol, the routing engine aggregation protocol is used to link the link estimation, data forwarding and the information management. Device driver is developed in the Linux kernel environment, the application program is used to access the kernel driver files in the API interface, in the host computer, QT development environment is established to achieve the development and integration of college educational management system. The simulation results show that the system has good network communication and data transmission capacity. In the university educational administration management, it can accept the control command for disposition parameter and carrying on the corresponding movement, the teaching management efficiency and the ability is enhanced.
\end{abstract}

\section{Introduction}

University educational administration management system is a complex and comprehensive system, and integrated university educational administration management system can achieve the teaching resource scheduling, teachers to optimize distribution, student information management, the classroom information management and remote network teaching function. With the continuous development of network information technology, educational management system has made a big progress with the computer network information technology. Through the optimization design of the educational management system, development of university educational administration management system and the network environment platform are obtained, the utilization efficiency of teaching resources is improved, and optimization scheduling and management of information resources in Colleges and universities is reached. By optimizing the design of university educational administration management system of network environment and network platform, the teaching, students, teachers and textbooks resources and matched and utilized reasonably. The tracking ability of information for students and teachers is improved, and promote the continuous upgrading of quality education. Therefore, optimization design method for the study of college teaching management system has obtained an important significance to improve the level of higher vocational education, and relevant system design method has obtained the great attention of people ${ }^{[1]}$.

University teaching management platform includes four basic entity objects: goal of educational management, teaching information processing nodes, teaching resources data collection node and network communication and information management platform ${ }^{[2]}$. A large number of college teaching information processing nodes are distributed in each user layer and are randomly deployed, through the self-organization of the network, collaborative formation of teaching resources are 
managed on information scheduling platform. Users and remote task management units are exchanged through the external network, such as local area network, campus network and GPRS communication network or Internet, and the educational administration is the core layer of interaction. The traditional educational administration network platform has flat structure, it is deployed in the monitoring area for data acquisition of the micro routing link layer homogeneous nodes, each node's computing ability, communication distance and energy supplies are about evenabout. The nodes data collection is completed by multi hop communication with other nodes in the network, forwarding data back to the sink node, by sink node and other network connection, remote access and network query and management are realized. Although it can work, but with the increase in the number of nodes, the expansion of network coverage, long communication path will lead to packet loss probability increases, the network performance is declined, and it also can cause more energy consumption for forwarding data of intermediate nodes, and reduce the network lifetime. An optimizing design of network development platform of university educational administration management system is proposed based on the routing engine. In the routing and data forwarding protocol, the routing engine aggregation protocol is used to link the link estimation, data forwarding and the information management. Device driver is developed in the Linux kernel environment, the application program is used to access the kernel driver files in the API interface. The simulation results show that the system has good network communication and data transmission capacity. In the university educational administration management, it can accept the control command for disposition parameter and carrying on the corresponding movement, the teaching management efficiency and the ability is enhanced. It shows good application value in practice.

\section{Overall design description and software architecture of the educational administration system}

\subsection{Overall design description and function analysis of the universities educational administration system}

Educational administration network platform has application support layer, the university educational administration management infrastructure platform and the educational administration management, and application service layer part of the common functions and management, such as information security and other composition of the university educational administration management network platform middleware and platform software ${ }^{[3-5]}$.

The basic implication is that the application of the support layer is used to support the application of the business layer for each application area. Services layer provides a variety of common services, in this layer, it is the core of the middleware software; management and information security is the base of all levels. The middleware and platform software architecture of educational administration network platform is divided into four levels: network adaptation layer, basic software layer, adaptation layer, application layer development and application adapter layer and basic software layer are composed in college educational management platform network node embedded software (deployed in educational administration management in universities network nodes platform) system structure. Development of application layer and the base layer of software composed the educational administration management network application platform support structure (Support business application development and implementation). These middleware constitute the public foundation of the platform software of the network platform, which provides a high degree of flexibility, modularity and portability. Architecture of network platform of educational administration management is shown in Figure 1. 


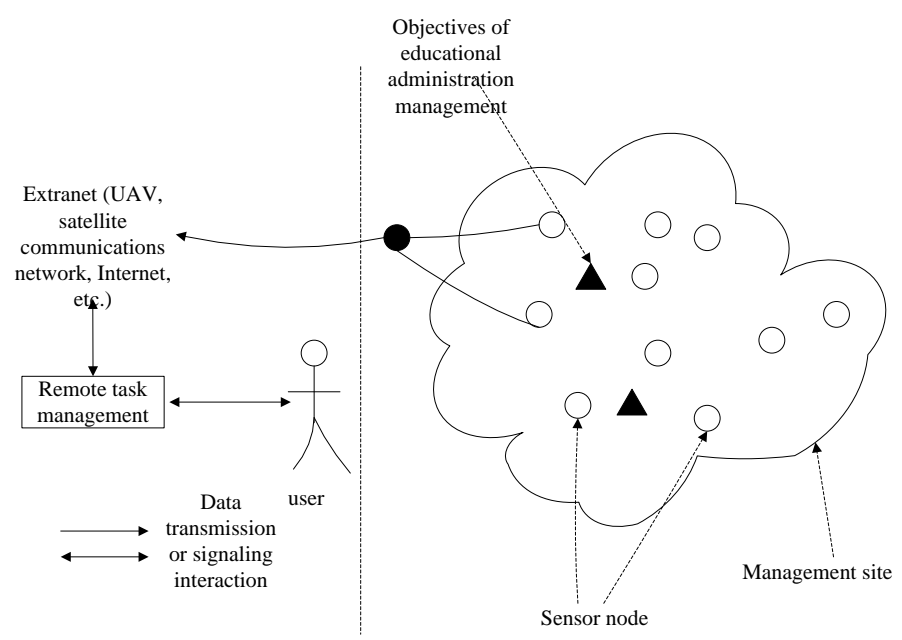

Figure 1. Architecture of network platform of educational administration management

The network platform of university educational administration management is divided into the following several middleware:

Network middleware: to complete works such as the access services, network generation services, network self-healing services, network connectivity services, etc.

Configuration middleware: to complete works such as routing configuration, the topology of the adjustment, etc. for the educational administration of the network platform.

Functional middleware: to complete the common function of various application services of the network platform of educational administration management in Colleges and universities, and provide a variety of functional framework interface.

Management Middleware: to achieve a variety of management functions, such as directory service, resource management, energy management, life cycle management, and so on.

Security middleware: to achieve a variety of security functions, such as security management, security monitoring, security audit

Educational administration network platform middleware and platform software uses hierarchical and modular architecture and makes it more adaptable to the requirements of educational administration management, the development and application is simple, and middleware technology is more simple and clearly to meet the application needs. On the one hand, middleware provides solution to meet the educational administration management in Colleges and universities network platform personalized applications, formed a particularly suitable supporting environment; on the other hand, middleware integrates the information and it can solve problems of software platform on educational administration management network platform, the middleware has flexibility and scalability performance to ensure network security and improve the educational administration management ability and energy efficiency of educational administration management. In order to reduce the complexity of the development and application, university educational administration management platform needs to achieve self-organization network technology, relative to general significance of ad hoc networks, routing link layer network have some characteristics as following:

(1) The number of nodes in the network platform of the educational administration management is big.

(2) The biggest characteristic of the self organizing routing link layer network is the energy confinement.

(3) The particularity of the environment of the routing link layer network application.

(4) Routing link layer node high density deployment, network topology change fast. Challenges to the maintenance of the topology are also presented.

Based on the analysis of the above characteristics, routing link layer network need adapts the characteristics of the network system structure according to the requirements of users on the network design, the standardization of network protocols and algorithms provide a unified technical specification, so that it can meet the user's demand. 


\subsection{Software architecture of the network development platform of the educational administration management system}

The communication architecture of network platform of educational administration management can be divided into horizontal communication protocol layer and vertical routing link layer network management. The communication protocol layer can be divided into physical layer, link layer, network layer, transport layer and application layer. And network management can be divided for energy management surface, mobility management surface and task management surface, face management exists is mainly used to coordinate the function of different levels in order to obtain a comprehensive consideration of the optimal design in terms of energy management, mobility management and task management.

(1) Physical layer. The transmission medium of the network platform can be the wireless, and cable medium. The network platform of the educational administration management mainly uses the wireless transmission.

(2) Data link layer. Data link layer is responsible for data stream multiplexing, data frame detection, media access and error control. The data link layer ensures the point to point to point connection of the educational administration management network platform.

(3) Network layer. Routing link layer network nodes are distributed in the environment or around the test environment, and a special multi hop wireless routing protocol is needed between the nodes in the routing link layer network and the receiving nodes.

According to the characteristics of the above analysis, the ARM software operating environment is established. Here we first compile the ARM platform based on the QWT Library (does not contain the Designer QWT Qt Control Library). First, download the source package, and extract to any directory. Edit the qwtconfig.pri file under the QWT directory, change the installation directory as shown in Figure 2.

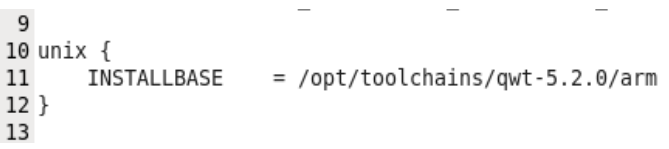

Figure 2. Edit file

Go to the directory to store the directory, enter the command install-qt-x11.sh source, start to compile, install. When you encounter authorization information, enter Yes. At the same time to comment out the following sentence, otherwise the compiler will be reported to the wrong:

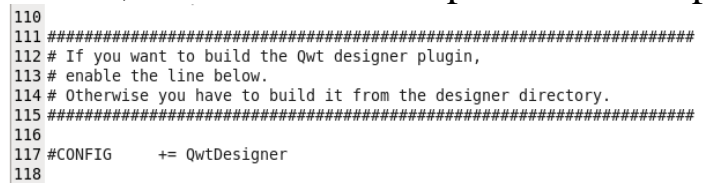

Figure 3. File Compilation

Run "make" command, start compilation, the compiler is completed, the LIB rootfs library runs into the /lib under the QWS. Compile link generates executable file which can be run on the target platform, and the software design of network development platform is realized.

\section{Routing engine aggregation protocol design and network development platform optimization}

In this paper, the routing engine aggregation protocol method is used to optimize the network design, and the convergence data communicates to the base station, it is a common requirement of routing link layer network applications. The common method is to establish at least one convergence tree, and the tree root node is used as the base station. When the data converges to the root node generated by the node, it only needs to go up along the convergence tree. When the node receives the data, it will be forwarded to other nodes, and finally can get to the root node. TinyOS2.x provides two kinds of aggregation protocols: CTP protocol and MultiHopLQI protocol, which can solve these problems well. If it needs to achieve new convergence protocol in TinyOS to 119 definition of compliance of TEP (TinyOS enhancement Proposals) CollectionC configuration specification, it 
mainly defines the aggregation protocol interfaces and components, network nodes are divided into four kinds of roles: sender, listener, intermediate and the receiver.

The software design of the system is constructed based on the Linux2.6.32 kernel. The embedded Linux system is composed of system boot loader, Linux2.6.32 kernel, file system and application program. On this basis, the configuration compile Linux kernel, modify the top of the Makefile file, this file is the entire kernel configuration, and compile the control file. First of all, to make the default Linux-2.6.32.2 target platform to become a platform for ARM. Modify the total directory Makefile. In the original document:

export KBUILD_BUILDHOST :=\$(SUBARCH)

ARCH $?=\$($ SUBARCH $)$

CROSS_COMPILE ?=

Modify as:

export KBUILD_BUILDHOST :=\$(SUBARCH)

ARCH $?=$ arm

CROSS_COMPILE ?=arm-linux-

Through the above process, the design of routing engine aggregation protocol and the development platform of network development platform are realized.

\section{Simulation experiment and result analysis}

In order to test the performance of the routing engine of the university educational administration management system designed in this paper, and test the performance of the network development platform, the simulation experiment is carried out. In the experimental process, device driver is developed in Linux kernel environment, application access device drivers in the kernel file API export. At first, the program is loaded, and the loading process is shown in Figure 4.

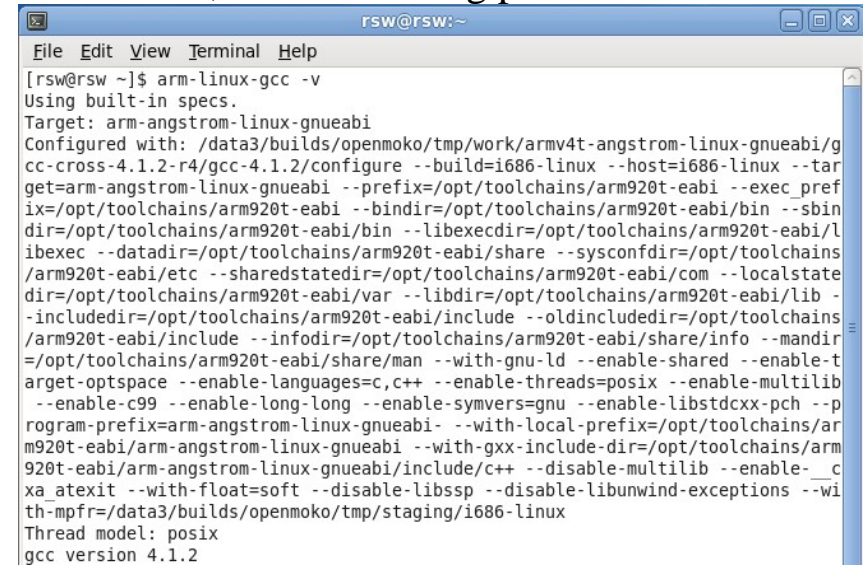

Figure 4. Program loading

Finally, the execution efficiency of the educational administration management system are tested as the index, and the simulation results are shown in Figure 5, it can be seen from the diagram, using the new method of network platform of university educational administration management system, it can improve the execution efficiency of the system, data communication transmission swallow throughput has been greatly improved, it shows the superiority of this method.

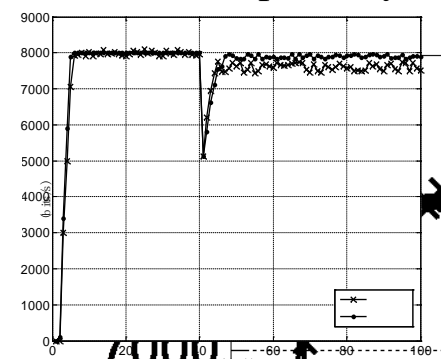

Figure 5. Performance testing 


\section{Conclusions}

The development of the network communication platform of university teaching management system is researched to improve the efficiency of college educational administration management, and promote the sharing and scheduling of academic resource information. Device driver is developed in the Linux kernel environment, the application program is used to access the kernel driver files in the API interface, in the host computer, QT development environment is established to achieve the development and integration of college educational management system. The simulation results show that the system has good network communication and data transmission capacity. In the university educational administration management, it can accept the control command for disposition parameter and carrying on the corresponding movement, the teaching management efficiency and the ability is enhanced.

\section{Acknowledgments}

Many thanks to the support from "Discussion of Educational Management System of Automatic Platform", NO.: GDJG20142535, Higher Education Teaching Reform Project of Guangdong province.

\section{References}

[1] CUN Yong-jun, ZHNAG Yong-hua. Linux System Dual Threshold Scheduling Algorithm Based on Characteristic Scale Equilibrium[J]. Computer Science, 2015,42(6):181-184.

[2] GAO Zhichun,CHEN Guanwei,HU Guangbo,et al. Fault Diagnosis and Optimal Data Clustering Based on K-Means with Slope Factor[J]. Computer \& Digital Engineering,2014,42(1): 14-18.

[3] LANG Zhen-hong.A design of self-study's platform system based on cloud computing[J].SAMSON,2016,(01):35-39.

[4] Yan Fang, Li Yuanzhang, Zhang Quanxin, Tan Yu'an. Object-Based Data De-Duplication Method for OpenXML Compound Files. Journal of Computer Research and Development, 2015, 52(7): 1546-1557.

[5] XING Changzheng, LIU Jian. Evolutionary data stream clustering algorithm based on integration of affinity propagation and density. Journal of Computer Applications, 2015, 35(7): 1927-1932. 